\title{
1 GPS Differential Code Biases determination: Methodology and Analysis
}

2 Jaume Sanz, J. Miguel Juan, Adrià Rovira-Garcia, Guillermo González-Casado

3 gAGE/UPC. Universitat Politécnica de Catalunya, Barcelona, Spain.

$4 \quad+34-93.401 .60 .30$

$5+34-93.401 .59 .81$

6 jaume.sanz@upc.edu

7 http://www.gage.upc.edu

8 DOI: $10.1007 / \mathrm{s} 10291-017-0634-5$

9 https://link.springer.com/article/10.1007\%2Fs10291-017-0634-5

10 Abstract We address two main problems related to the receiver and satellite Differential 11 Code Biases (DCBs) determination. The first issue concerns the drifts and jumps experienced 12 by the DCB determinations of the International GNSS Service (IGS) due to satellite 13 constellation changes. A new alignment algorithm is introduced to remove these nonphysical 14 effects, which is applicable in real-time. The full-time series of 18 years of Global Positioning System (GPS) satellite DCBs, computed by IGS, are realigned using the proposed algorithm. The second problem concerns the assessment of the DCBs accuracy. The short- and long-term receiver and satellite DCB performances for the different Ionospheric Associate Analysis Centers (IAACs) are discussed. The results are compared with the determinations computed with the two-layer Fast Precise Point Positioning (Fast-PPP) ionospheric model, to assess how the geometric description of the ionosphere affects the DCB determination and to illustrate how the errors in the ionospheric model are transferred to the DCB estimates. Two different determinations of DCBs are considered: the values provided by the different IAACs and the values estimated using their pre-computed Global Ionospheric Maps (GIMs). The second determination provides a better characterization of DCBs accuracy, as it is confirmed when analyzing the DCB variations associated with the GPS Block-IIA satellites under eclipse conditions, observed mainly in the Fast-PPP DCB determinations. This study concludes that the accuracy of the IGS IAACs receiver DCBs is approximately 0.3-0.5 ns and $0.2 \mathrm{~ns}$ for the Fast-PPP. In the case of the satellite DCBs, these values are about $0.12-0.20$ ns for IAACs and 0.07 ns for Fast-PPP.

Keywords: DCB, Ionospheric models, GPS, GNSS. 
Timing biases between $P_{1}$ and $P_{2}$ code measurements are referred to as inter-frequency biases or $P_{1}-P_{2}$ Differential Code Biases (DCBs). These hardware delays are embedded in both Global Positioning System (GPS) satellites and receivers, depend on the signal modulation and, like the ionospheric delay, on the frequency transmission. On the other hand, because the GPS Control Segment provides the satellite clocks relative to the ionospheric-free linear combination of $P_{1}$ and $P_{2}$ codes (IS-GPS-200H 2014)

$$
P_{I F}=\left(f_{1}^{2} P_{1}-f_{2}^{2} P_{2}\right) /\left(f_{1}^{2}-f_{2}^{2}\right)
$$

single-frequency users must compensate for these code biases, $P_{1}-P_{2}$ (Ray et al. 2005). Thus, an accurate determination of the satellite DCBs is needed for users applying ionospheric corrections.

Satellite DCBs can be calibrated in the factory within an anechoic chamber, but their values could change with time. Therefore, these biases shall be estimated in orbit from dualfrequency signals. In this manner, the DCBs and ionospheric delays can be derived from the geometry-free combination of code measurements

$$
P_{G F}=P_{2}-P_{1}
$$

which is only sensitive to the frequency-dependent delays, being modeled as constant or nearly constant parameters (Juan et al. 1997, Colleen et al. 1999).

Since June 1, 1998 the Global Ionospheric Maps (GIMs) and DCBs have been routinely estimated by the International GNSS Service (IGS) (Hernández-Pajares 2009). Several Ionospheric Associate Analysis Centers (IAACs) are involved in this IGS project, including the Centre for Orbit Determination in Europe (CODE; Berne, Switzerland), the Jet Propulsion Laboratory (JPL; Pasadena, CA, USA), the European Space Agency (ESA/ESOC; Darmstadt, Germany), and the Universitat Politècnica de Catalunya (UPC/IonSat; Barcelona, Spain). A weighted average of the individual determinations from the IAACs is computed to generate the combined IGS product, which is provided daily in Ionosphere Map Exchange (IONEX) format (Schaer et al. 1998).

An assessment of IGS DCB estimates is summarized in Hernández-Pajares et al. (2009), where typical $P_{1}-P_{2}$ DCB values in the range of -4 to $5 \mathrm{~ns}$ for the satellites are found, with discrepancies between IAACs at the level of a few tenths of a nanosecond, while the 
receiver DCBs are usually from -20 to $15 \mathrm{~ns}$, with discrepancies up to a few nanoseconds.

Despite the above numbers for the DCB estimates, which refer to an inter-center comparison, and other results from similar performance indicators given in the literature (Montenbruck et al. 2014), the calibration of the DCBs accuracy is still an open problem. Trends and instabilities are observed in the estimated values, being necessary to discriminate between real effects and those related to the mis-modeling of the ionospheric model. From physical considerations, it is assumed that DCBs are constant, or almost constant parameters, and sensitive to the thermal conditions (Yue et al. 2011, Zhong et al. 2016). However, the time series of the DCBs from the IGS products exhibits jumps and long-term drifts, which are produced by changes in the satellite constellation (Schaer 2008).

As it is well known, the DCBs estimation process is rank deficient and a reference value must be taken to remove the singularity. The IGS IAACs use the mean value of DCBs for all satellites, which is set to zero by convention. As indicated in Schaer (2008), the jumps mentioned above and the drifts correspond to changes in the satellite set used to compute the reference value, following the IGS convention. Moreover, due to the correlation with the ionospheric model, the estimated DCBs usually experience "pseudo" variations linked to the solar cycle and seasonal ionospheric effects (Zhang et al. 2014).

We provide some insight into the DCB accuracy calibration, discuss the key elements affecting their determinations and separate physical and non-physical phenomena affecting their stability and time evolution.

In the next sections, we present two different strategies for the DCB estimation and then we discuss the alignment problem mentioned above for the DCB time series computed by the IGS IAACs. We propose a new alignment procedure which is immune to satellite constellation changes. Once these topics have been addressed, we define a metric to analyze the performance of the receiver and satellite DCBs computed by the different IAACs for the year 2014. This analysis involves the DCB values estimated using the two strategies and it is conducted by considering two temporal scales: short-term (daily repeatability) and long-term (annual stability). Finally, satellite Block-IIA DCB variations under eclipse condition are detected and are used to cross-check the performance of previous methods and asses the accuracy of DCB estimates. A summary and conclusions are given in the last section. 


\section{Strategies for DCB estimation}

93 It is well known that the ionospheric effects and the DCB effect are interrelated, and must, therefore, be decorrelated. In fact, the accuracy of the DCB determinations is closely linked to the performance of the ionospheric model used. Thus, the geometric description adopted for the ionosphere, such as one-layer or multi-layer, or the layer height assumptions, affects the estimation of both satellite and receiver DCBs (Hernández-Pajares et al. 1999, Komjathy et al. 2002). Other aspects are the data processing approach influencing the results, e.g. the time update, the process noise used in the Kalman filter or the base functions used.

Regardless of the characteristics of the ionospheric model adopted, two main strategies are currently applied to estimate the DCBs:

\section{i) Common adjustment of DCBs and GIM}

103

This strategy consists of estimating the DCBs and the parameters of the ionospheric model in a common adjustment process. The input data are the geometry-free combination of carrier phases $\left(L_{\mathrm{GF}}=L_{1}-L_{2}\right)$, leveled with the corresponding $P_{\mathrm{GF}}$ code, as it is done by CODE, JPL and ESA IAACs (Li et al. 2012) or with the ambiguities fixed PPP, as in the Fast Precise Point Positioning (Fast-PPP) estimates from the Research group of Astronomy and Geomatics (gAGE) (Rovira-Garcia et al. 2015). These DCBs are provided by the IAACs and gAGE in IONEX files. Hereinafter, we will refer to these DCBs as the "reported DCBs."

ii) DCBs estimation from a pre-computed GIM

111 This strategy consists of estimating the DCBs using a previously computed ionospheric

112 model. Indeed, the DCBs are estimated by subtracting the ionospheric model predictions to 113 the geometry-free combination of pseudoranges $P_{\mathrm{GF}}$. This is the approach applied by the UPC 114 IAAC (Hernández-Pajares et al. 2009, Montenbruck et al. 2014). We will re-estimate the 115 reported DCBs from the IAACs and gAGE following this strategy. From now on, we will 116 refer to them as the "re-estimated DCBs."

117 One advantage of strategy (ii) is that the same GIM estimated from a given satellite 118 constellation, such as GPS, can be used to estimate the DCBs for any other constellation of 119 satellites. Moreover, the DCBs can be estimated with any time update. In fact, the sub-daily 120 stability of the DCBs computed with strategy (ii) is used in Rovira-Garcia et al. (2016) to 121 compare the performances of different ionospheric determinations. 
In principle, both strategies should give similar DCB results, if the same ionospheric model, estimated jointly with the DCBs by the strategy (i), is used in strategy (ii). But the

124 GIMs derived from strategy (i) usually have gaps in regions where receivers are not 125 available, such as in ocean areas, which are filled by an interpolation scheme to generate the 126 final GIM. Other constraints can also be applied in the DCBs to give more strength to these 127 parameters adjustment. All these conditions can affect the DCB estimates when assessed 128 using strategy (ii). For instance, in the case of Fast-PPP, no constraints are introduced in the decorrelation of DCBs and the ionosphere, but an interpolation scheme is used to fill the gaps when building up the final GIM. This can produce some discrepancies in DCB estimates when assessed using strategy (ii). In the case of the IAACs applying strategy (i), such constraints are not well detailed, and thence, strategy (ii) provides a complementary determination of the DCBs that is more traceable regarding ionosphere and DCBs decorrelation.

\section{DCB alignment problem}

137 As mentioned in the introduction, the DCBs should be constant or almost constant. However, the time series of daily GPS DCB values of the IGS Final Product IONEX files presents jumps and drifts, as reported by several authors (Schaer 2008, Zhang et al. 2014). This effect is due to changes in the satellite constellation, as illustrated in Figure 1 (black points), where the vertical lines indicate epochs having satellite exclusions (dashed line) or incorporations (solid line) to the DCBs alignment process. These epochs are also summarized in Table 1. In this figure, the DCBs of the different satellites are shifted to zero in the first day to remove the different initial biases and better see the group evolution.

An explanation of the apparent increase in the DCB values observed in Figure 1 can 146 be inferred from Table 1. From this information, it follows that the DCB values of new 147 Block-IIF satellites have larger negative DCB values than the values of Block-IIA satellites. 148 Indeed, they are responsible for the positive jumps experienced when they are incorporated in 149 the computation. That is, when the new satellites of Block-IIF are included in the 150 computation, the mean DCB value, used by IGS as alignment reference, decreases and thus contributes to the ascending drift. This effect is smaller when the Block-IIA satellites are removed from the computation, as their values are closer to the mean value. 


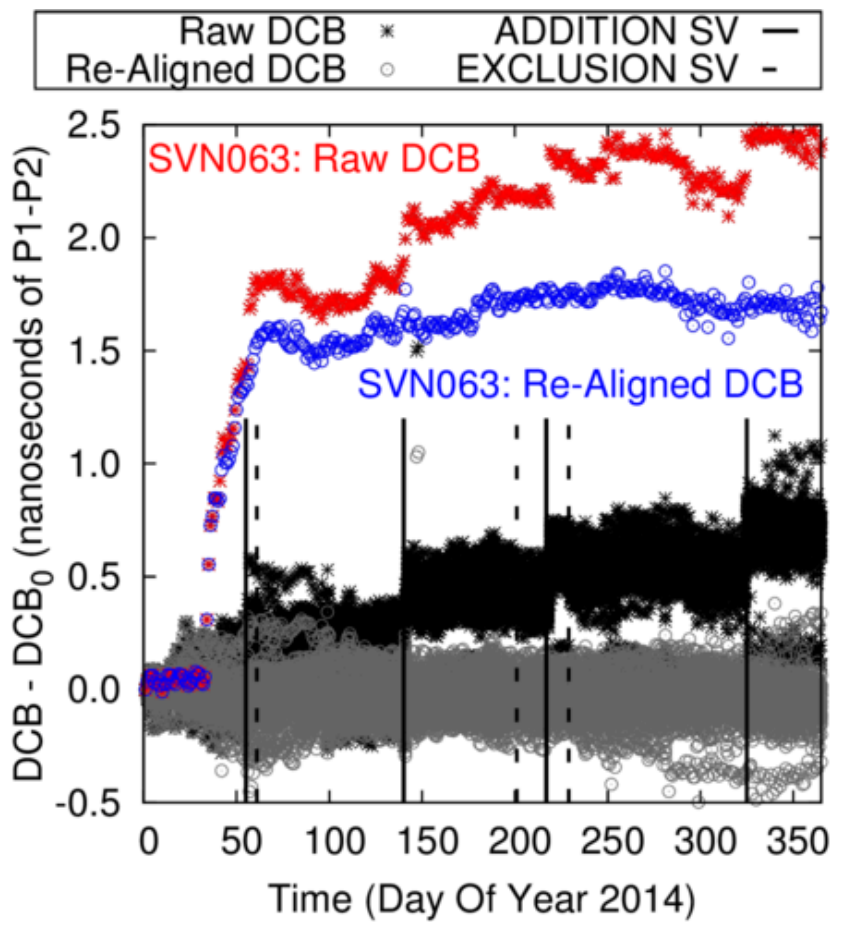

155 Fig. 1: Effect of using different references to align the IGS Final Product DCBs. The plot depicts the time evolution of satellite DCB estimates for all satellites during the year 2014: i)

157 The black stars show the DCBs of satellites aligned with the mean value of all satellites available at each epoch, i.e. the IGS convention. ii) The gray circles show the same DCBs but aligned with a common set of satellites established for the entire year of 2014. iii) The colored isolated trend of points corresponds to the anomalous satellite SVN063 (PRN01).

161 Vertical lines indicate satellite incorporation (solid) and exclusion (dotted), see Table 1. Note: SVN063 was not included in the satellite set used in (ii).

163

A solution proposed by Schaer (2008) to remove these apparent jumps and drifts was to realign the time series by using a fixed common set of satellites, established for the entire period of study. This approach corresponds to the gray circles in Figure 1. As expected, the drift disappears for all satellites having nominal behavior. In this manner, an actual physical anomaly experienced by Space Vehicle Number 63 (SVN063) is more clearly evidenced in the figure. This happened between Day of Year (DoY) 34 and 60, degrading the DCB estimates. NANU 2014027 was issued on March 2014, setting the satellite SVN063 (PRN01) to unusable until further notice (http://celestrak.com/GPS/NANU/2014/ nanu.2014027.txt). It

172 is worth noting that anomalies affecting any satellite involved in the average used to align the DCBs will contaminate the results of the others if not removed. 
174 Table 1: Changes in the GPS satellite constellation used by IGS to align the DCBs. The 175 Exclusion and Incorporation columns indicate the Day of Year (DoY) 2014 when the satellite 176 is incorporated or excluded in the average. The last column indicates the DCB value.

\begin{tabular}{|c|c|c|c|c|c|}
\hline Block & SVN & PRN & $\begin{array}{c}\text { DoY } \\
\text { Decommission }\end{array}$ & $\begin{array}{c}\text { DoY } \\
\text { Exclusion }\end{array}$ & $\begin{array}{r}\text { DCB } \\
\text { (ns) }\end{array}$ \\
\hline \multirow{4}{*}{ IIA } & 036 & 06 & 052 & 061 & -2.18 \\
\hline & 039 & 09 & 139 & 201 & -1.26 \\
\hline & 033 & 03 & 214 & 229 & -2.17 \\
\hline & 038 & 08 & 303 & 102 (2015) & -2.17 \\
\hline & SVN & PRN & $\begin{array}{c}\text { DoY } \\
\text { Launch }\end{array}$ & $\begin{array}{c}\text { DoY } \\
\text { Incorporation }\end{array}$ & $\begin{array}{l}\text { DCB } \\
\text { (ns) }\end{array}$ \\
\hline \multirow{4}{*}{ IIF } & 064 & 30 & 052 & 055 & -7.14 \\
\hline & 067 & 06 & 137 & 140 & -7.71 \\
\hline & 068 & 09 & 214 & 217 & -5.19 \\
\hline & 069 & 03 & 302 & 325 & -5.91 \\
\hline
\end{tabular}

Obviously, a solution based on using such a fixed common set of satellites established 180 for the entire period of study cannot be applied in an operational mode for the daily DCB 181 estimates, as it is not possible to predict the variations in the satellite constellation over time.

182 Nevertheless, because the trend in the IGS-aligned DCBs is common for all satellites, it can 183 be removed by aligning the daily solutions with the mean value computed with a common set 184 of satellites between the current day and some previous days $\left(N_{D}\right)$, e.g. the previous week, 185 where any anomalous satellite shall be excluded from this average.

186 In this manner, a constellation change, i.e. as a result of a satellite launch or 187 decommission, will not vary the reference, where, on the other hand, the averaging over a 188 given number $N_{D}$ of previous days is done to strengthen results.

189 Hence, the realignment procedure is as follows: 
192

i. Outliers are removed by excluding satellites experiencing DCB jumps greater than 50 centimeters (1.7 ns). These jumps are computed for each satellite, as the difference between the current and the averaged value over the last $N_{D}$ days (we take $N_{D}=7$ ).

ii. A common satellite set of $N_{S}$ satellites for the current day and the last $N_{D}$ days is established.

iii. From DCB values $\left(X^{j}\right)$, the mean values for the current day $\left(\bar{X}_{C}\right)$ and the $N_{D}$ previous days $\left(\bar{X}_{P}\right)$ are computed over the common satellite set:

$$
\bar{X}_{C}(n)=\frac{1}{N_{s}} \sum_{j=1}^{N_{S}} X^{j}(n) \quad \text { and } \quad \bar{X}_{P}(n)=\frac{1}{N_{D}} \sum_{i=1}^{N_{D}} \frac{1}{N_{s}} \sum_{j=1}^{N_{S}} X^{j}(n-i)
$$

where $n$ is the given day.

iv. The DCBs of all satellites, including uncommon satellites, are corrected with the difference between the two mean values computed in the previous step,

$$
\delta(n)=\bar{X}_{C}(n)-\bar{X}_{P}(n) \text {. }
$$

After some algebraic manipulations, this difference can be written as:

$$
\delta(n)=\frac{1}{N_{D} N_{s}} \sum_{i=1}^{N_{D}} \sum_{j=1}^{N_{S}}\left[X^{j}(n)-X^{j}(n-i)\right]
$$

Applying this procedure, the DCBs of common satellites do not experience artificial variations due to changes in the satellite constellation. New satellites are incorporated into the mean calculation with $N_{D}$ days of delay, but this does not cause any jumps, unlike the IGS convention.

It must be noted that this alignment procedure gives the same results as the conventional IGS procedure when no changes occur in the satellite constellation. Indeed, when $N_{D}=1$ and no changes take place in the satellite constellation, then $\delta(n) \equiv 0$. 

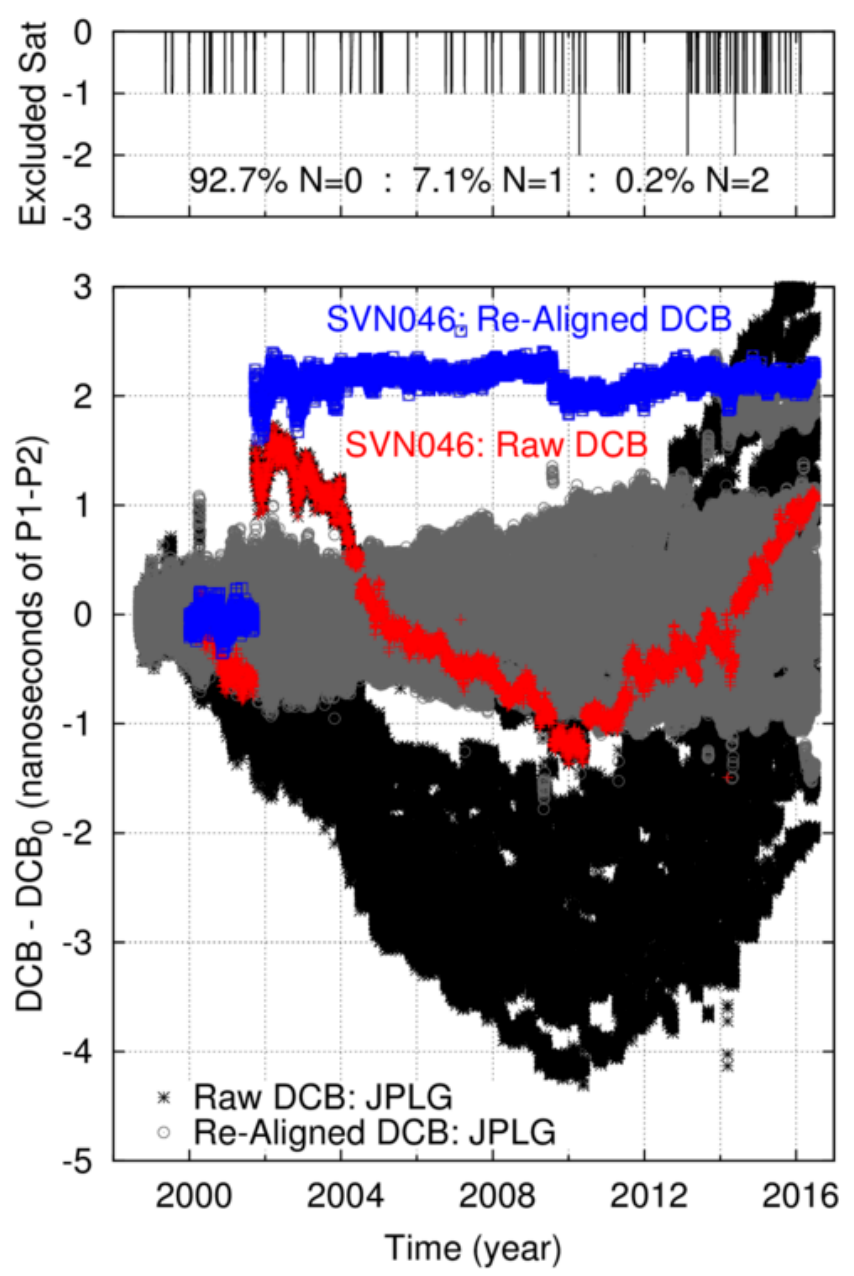

213 Fig. 2: Evolution of satellite DCBs (JPLG Final Product) from 1998 to 2016. In the bottom 214 panel: i) The black stars show the DCBs aligned with the mean value of all satellites available 215 at each epoch, i.e. the IGS convention. ii) The gray circles show the same DCBs but aligned 216 with the new alignment procedure proposed in this research. The value of the DCB for 217 SVN046 (PRN11) is highlighted in both approaches. The top panel depicts the number of 218 satellites discarded to compute the mean value of DCBs over the previous 7 days.

Figure 2 illustrates the performance of this new alignment procedure for the JPLG 221 Final Product DCBs over 18 years and compares the behavior with DCBs aligned following 222 the IGS convention, i.e. the values read from the IONEX files. These values are shifted to zero in the first epoch to better depict the group evolution. As shown in the bottom panel, the artificial drift appearing in the IGS conventional alignment, due to constellation changes, is eliminated by the new method. Thus, thanks to this alignment process, it is possible to detect a jump in the re-aligned DCB of SVN046 (PRN11), in blue, near the end of 2009 (DoY 213), which cannot be identified in the raw DCB, in red, due to the constellation jumps. This figure 
also highlights a bigger DCB jump experienced by SVN046 at the beginning of time series (DoY 256 of the year 2001). NANU 2001120 was issued on this day (see http://celestrak.com/GPS/NANU/2001/nanu.2001120.txt).

The top panel of Figure 2 shows the number of satellites discarded in the common set selection. As can be observed, in $92.7 \%$ of the time the same number of satellites is used by the IGS convention and the proposed re-alignment method, and thence both alignment procedures are equivalent over these periods. Additionally, in $7.1 \%$ of the time there is a single satellite discrepancy caused by the 1 -week buffer. Only in the remaining $0.2 \%$ of the time there are two satellites less.

\section{Performance assessment of DCB estimates}

Once the alignment problem has been fixed with the proposed method, this section will focus on assessing the capacity of the estimation process to de-correlate the DCBs from the ionosphere. The goal is to calibrate the accuracy of the DCB estimates and to identify the level of physical anomalies that can be detected.

Data set

The DCB values from the IONEX files, i.e. the reported ones provided by the different IGS IAACs throughout the entire year of 2014, realigned with the procedure given in this work, will be used. The following solutions are taken: IGS Combined Final Product (IGSG), CODE Final Product (CODG), JPL Final Product (JPLG) ESOC Rapid Product (EHRG), and UPC Rapid Product (UQRG). The time update of the GIMs is 2 hours for the Final Product, 1 hour for the ESOC Rapid Product, and 15 minutes for the UPC Rapid Product. These data files have been selected to have a wide sample of products involving different IAACs, time updates, and latencies.

The DCBs computed by the Fast-PPP (FPPP) ionospheric model with a 15-minute sampling rate will also be included in the data set to compare with the estimates from a model having a different geometry, i.e. two-layer instead of a single-layer grid.

Besides the reported DCBs of the IONEX files, an additional set of re-estimated 
combination of unambiguous carrier phase measurements after subtracting the STEC from the precomputed GIM associated with the same IONEX files. The unambiguous carrier phases, see equation (2) in Rovira-Garcia et al. (2016), are used instead of code-leveled carriers to avoid contamination from code measurement noise. Notice that this code noise would increase the standard deviation by some tenths of one nanosecond.

\section{DCB Performance Metrics}

In nominal conditions, it can be assumed that satellite and receiver DCBs are stable over time. Therefore, except for actual physical effects, e.g. thermally induced variations, or anomalous behaviors, the lack of stability would be a consequence of the mis-modeling of the ionospheric model. Hence, the main question is whether a worsening in the DCBs is a true physical effect or whether ionospheric modeling errors induce it.

Two different temporal scales will be considered in this study as the metric for the DCB assessment: a short-term scale, where the "Daily Repeatability" is used to analyze the daily self-consistency of the estimates, and a long-term scale to characterize their "Annual Stability" and analyze how the ionospheric mis-modeling is transferred to the DCBs over the year.

\section{Daily Repeatability}

The day-to-day variations are taken as a measure of DCB repeatability, i.e. the consistency of two independent solutions computed for two consecutive days when the ionosphere is expected to be similar. As the GPS geometry repeats daily, similar mis-modeling is experienced in two consecutive days. This statement is illustrated in Figure 3, which shows the DCB values of IGSG and FPPP re-estimated using the pre-computed GIMs. As depicted in this figure, the variations of the 1-hour independent batch estimates are larger than the dayto-day estimates from 24-hour batches. That is, the error of sub-daily, i.e. hourly, estimates evolve over the day as the satellite geometry and the electron content changes, but after 24 hours, these variations are mostly compensated for. Hence, the Daily Repeatability performance indicator, calculated as the RMS of the Day-to-Day variations over an entire year, provides a "lower bound of the DCB estimation error" or, in other words, the level of actual anomalies that can be detected. 
Special care must be taken to ensure that the DCBs are estimated each day independently. For instance, as indicated in the header of the IONEX files of CODE, this IAAC applies a 3-day solution, which means that $2 / 3$ of the data is shared every two consecutive days, which results in smoothed DCBs, as will later be shown.

293

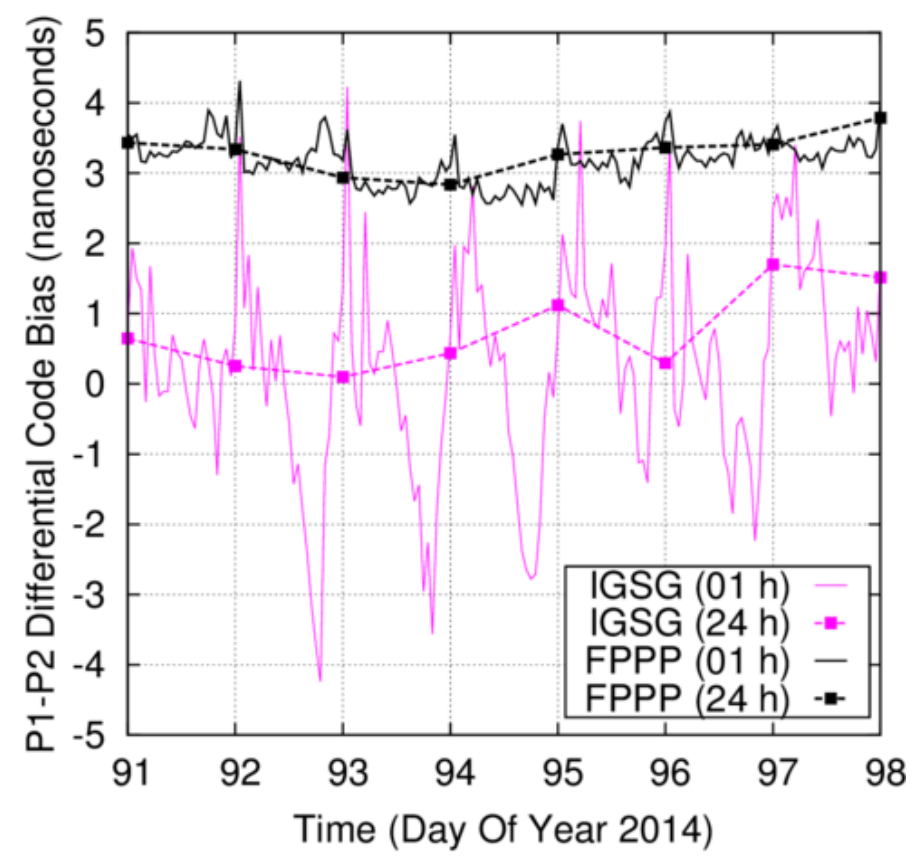

294 Fig. 3: Comparison of re-estimated receiver DCB estimates from a 1-hour batch (solid lines) and from a 24-hour batch (points and dashed lines). The results from the precomputed GIMs of IGS Final Product (IGSG) and Fast-PPP (FPPP) are shown in pink and black colors, respectively. These results correspond to the receiver CRO1 located at $17^{\circ} \mathrm{N}$ and $64^{\circ} \mathrm{W}$.

Annual Stability

301 The variation of the DCB value over longer periods, e.g. one year, is taken as a measure of its annual stability, being a performance indicator computed as the standard deviation of the daily DCB values over an entire year. Indeed, it is expected that the seasonal variations of the ionosphere or satellite eclipse conditions, among others, have an impact on the DCB estimations.

It is worth nothing that accumulated drifts, seasonal patterns or other long-term anomalies are not sampled by the day-to-day variations. However, long-term variations can 
be captured by the standard deviation of the time-series over a longer period, e.g. one year. See Figures 7 and 8 discussed later.

311 Results

312 The results of receiver and satellite DCB assessment are presented in this section, using the 313 previously defined metrics of Daily Repeatability and Annual Stability.

314 As is well known, the receiver DCBs are less stable than the satellite ones 315 (Hernández-Pajares et al. 2009). The reason for this different behavior is depicted in Figure 4. 316 As can be seen, the receiver DCBs are estimated from measurements of a small region of the 317 ionosphere, typically less than $20^{\circ} \times 20^{\circ}$, around the receiver location, as shown by the 318 Ionospheric Pierce Point (IPP) tracks in blue. On the contrary, the satellite DCBs are computed from measurements covering approximately half of the hemisphere, as shown by the IPP tracks in red. Then, local ionospheric mis-modeling directly affects the decorrelation

321 of receiver DCBs from the ionosphere, mainly in the sub-daily estimation (Figure 3), while in the case of satellites, it is compensated by the wider geographical coverage.

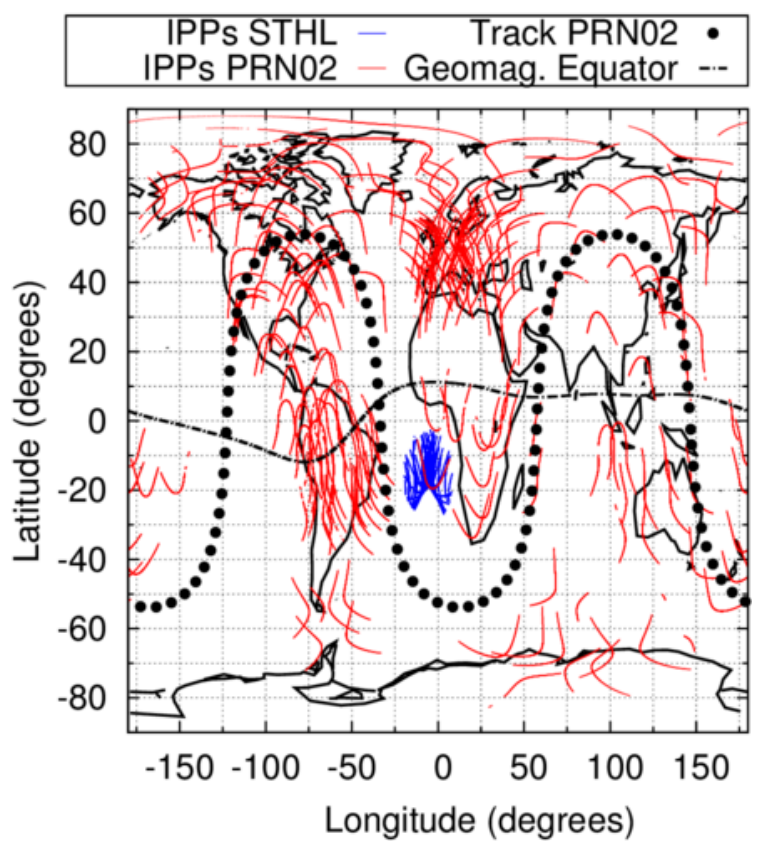

Fig. 4: Ionospheric Pierce Points (IPPs) footprints associated with the station DCB estimates for station STHL (16S, 6W) (blue) and with the satellite PRN02 estimates (red). Black points indicate the 24-hour satellite track. The constellation corresponds to GPS satellites on DoY 082, 2014. Dashed dot line indicates the geomagnetic equator. 
329 The reported DCB estimates of the different IGS IAACs are shown in Figure 5 for the entire 330 year of 2014 for two GPS receivers at low latitude, CRO1 (17N,64W), and mid latitude, 331 ZECK $(43 \mathrm{~N}, 41 \mathrm{E})$. Notice that these DCBs have been also realigned applying the realignment 332 procedure explained above, to remove the artificial jumps and drifts due to the satellite constellations changes. As can be observed, two well-defined peaks appear around the equinoxes for the one-layer model estimates. Figure 6 gives some insight into the source of this pattern. In this figure, the results of the consistency test, defined in Rovira-Garcia et al. (2016) to assess the accuracy of the ionospheric corrections, are shown for the same IAACs as in Figure 5. These results are depicted in Figure 6 for the entire year of 2014 as a function of time (top panel) and as a function of the geographic latitude (bottom panel).

Comparing Figures 5 and 6, it can be seen that the seasonal oscillations experienced by the DCBs are linked to the seasonal mis-modeling of the ionospheric estimates. Indeed, this example illustrates the effect of the correlations between both determinations, evidencing how the ionospheric error is transferred to the receiver DCB estimates.

The similar pattern found in the DCB determinations of the different IGS IAACs is not surprising, as the capability to de-correlate the DCBs from the STEC is strongly dependent on the geometrical description of the ionosphere used. As commented before, all IAACs de-correlate DCBs from the ionosphere using a one-layer ionospheric model at 450 $\mathrm{km}$ in height. Then, although different basis functions, such as spherical harmonics, voxels, interpolation schemes, e.g. splines and kriging, or time updates, e.g. 2 h, 1 h, and 15 min, are used, all of these determinations are affected by the mis-modeling associated with the onelayer model. This is the reason for the agreement between the determinations of the different IAACs reported in Hernandez-Pajares (2009).

The reported DCBs from the Fast-PPP ionospheric model have been included in the panels of Figures 5 and 6 to compare with a model having different geometry, i.e. the twolayer grid. As shown, the Fast-PPP estimates are more stable, and the equinox signature is the most difficult to appreciate. 

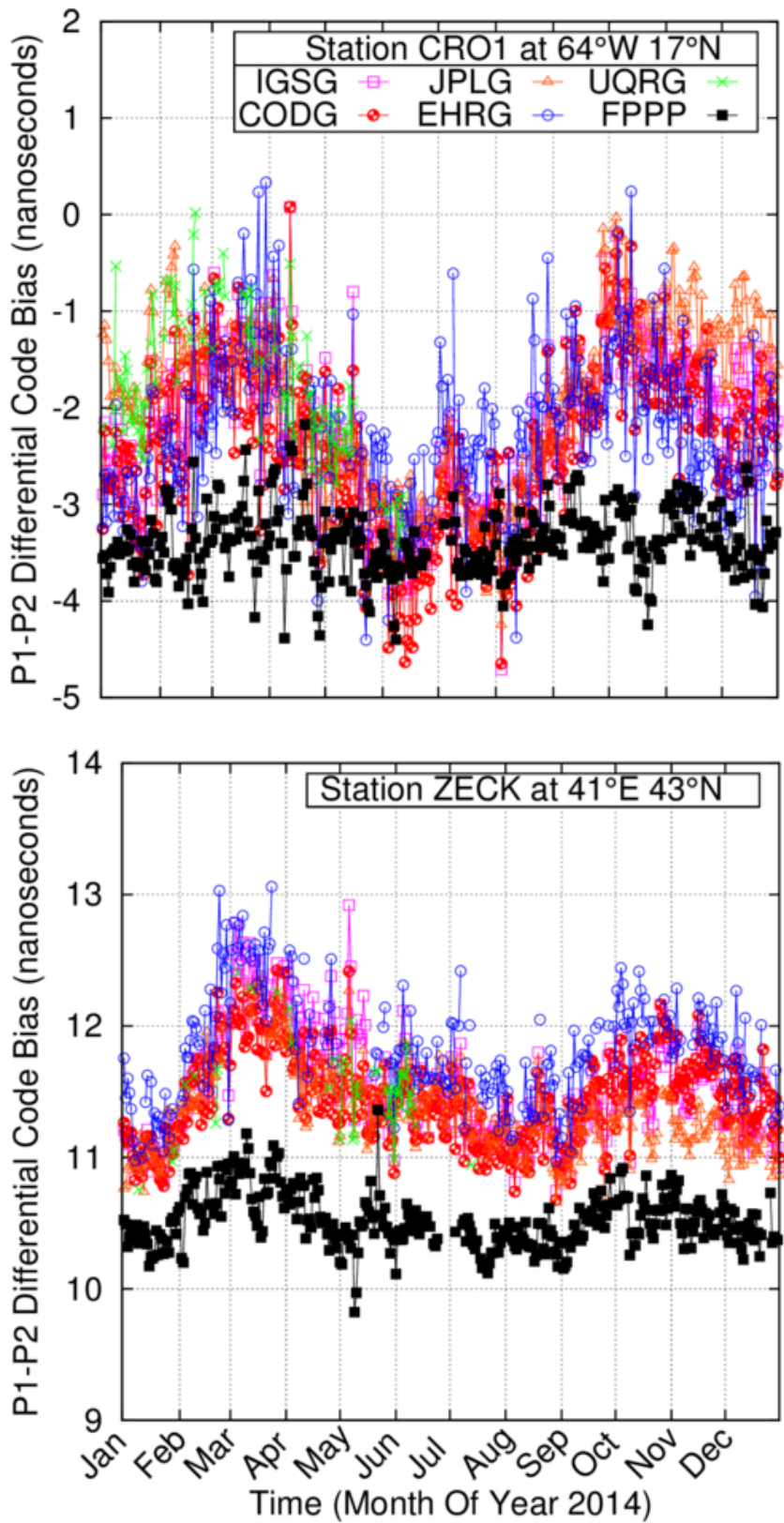

358 Fig. 5: Reported Receiver DCB estimates as a function of time for the entire year of 2014 for 359 the low-latitude receiver CRO1 $(64 \mathrm{~W}, 17 \mathrm{~N})$ (top panel) and the mid-latitude receiver ZECK 360 (41E,43N) (bottom panel). The estimates correspond to the IGS Combined Final Product 361 with 2 h Time-UPdate (TUP) (IGSG, pink squares), CODE with 2h TUP (CODG, red 362 circles), JPL with 2 h TUP (JPLG, orange triangles), ESOC with 1h TUP (EHRG, blue 363 circles), UPC with 15 min TUP (UQRG, green crosses) and Fast-PPP with 15 min TUP 364 (FPPP, black squares). 

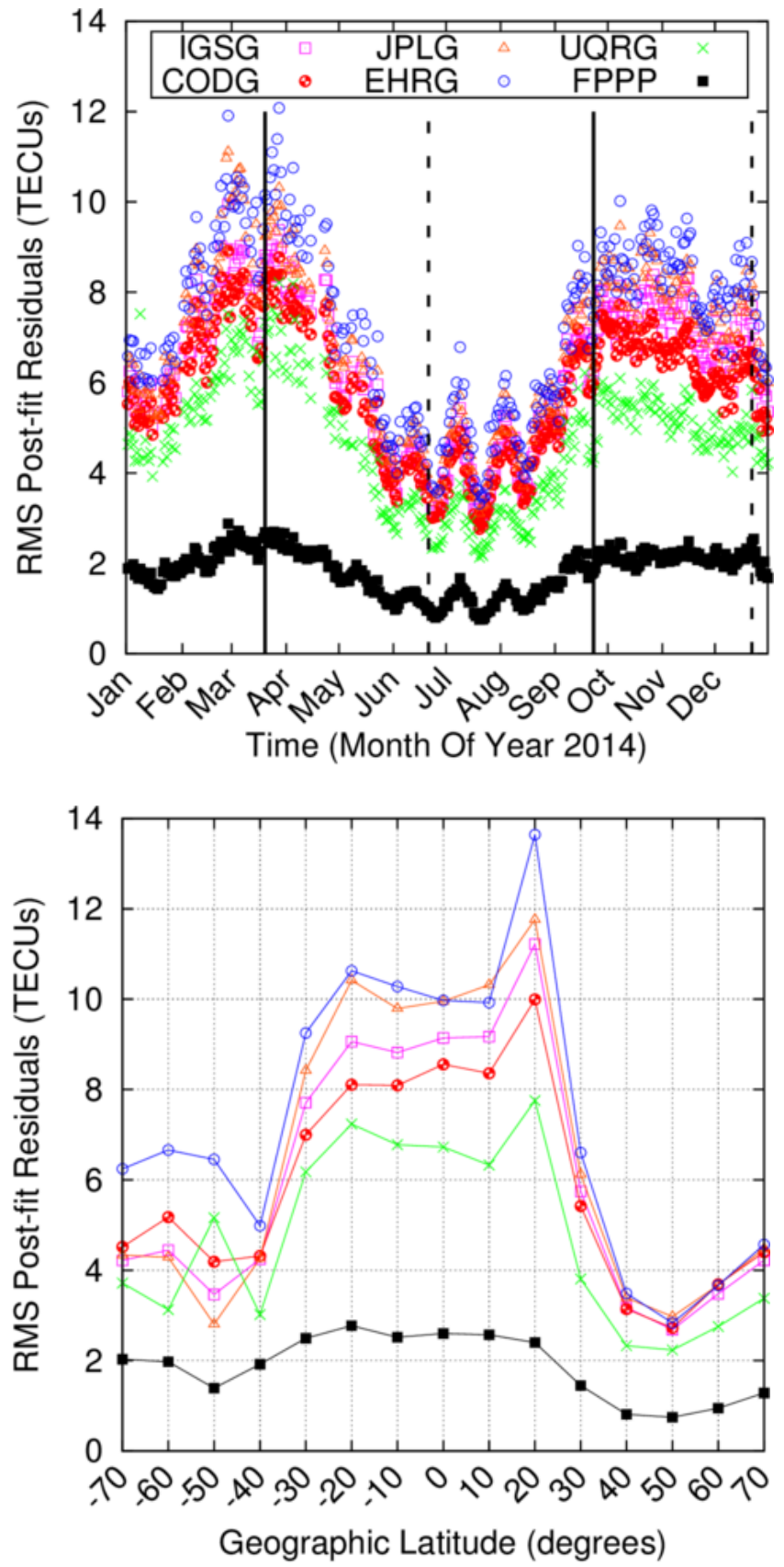

367 Fig. 6: Results of the consistency test among different global ionospheric models throughout 2014 as a function of time (top panel) and as a function of the geographic latitude (bottom panel). The same products as in the previous Figure 5 are shown. (bottom row) as a function of the receiver latitude. Each point in the panels corresponds to an 373 individual station, the name and coordinates of which are given in Table 2. Only stations used 374 by more than one IAAC and having values for more than 300 days in 2014 have been 375 included to guarantee a homogeneous comparison between IAACs. 

the IONEX files header. The right-hand panel shows the re-estimated DCBs.

378

379
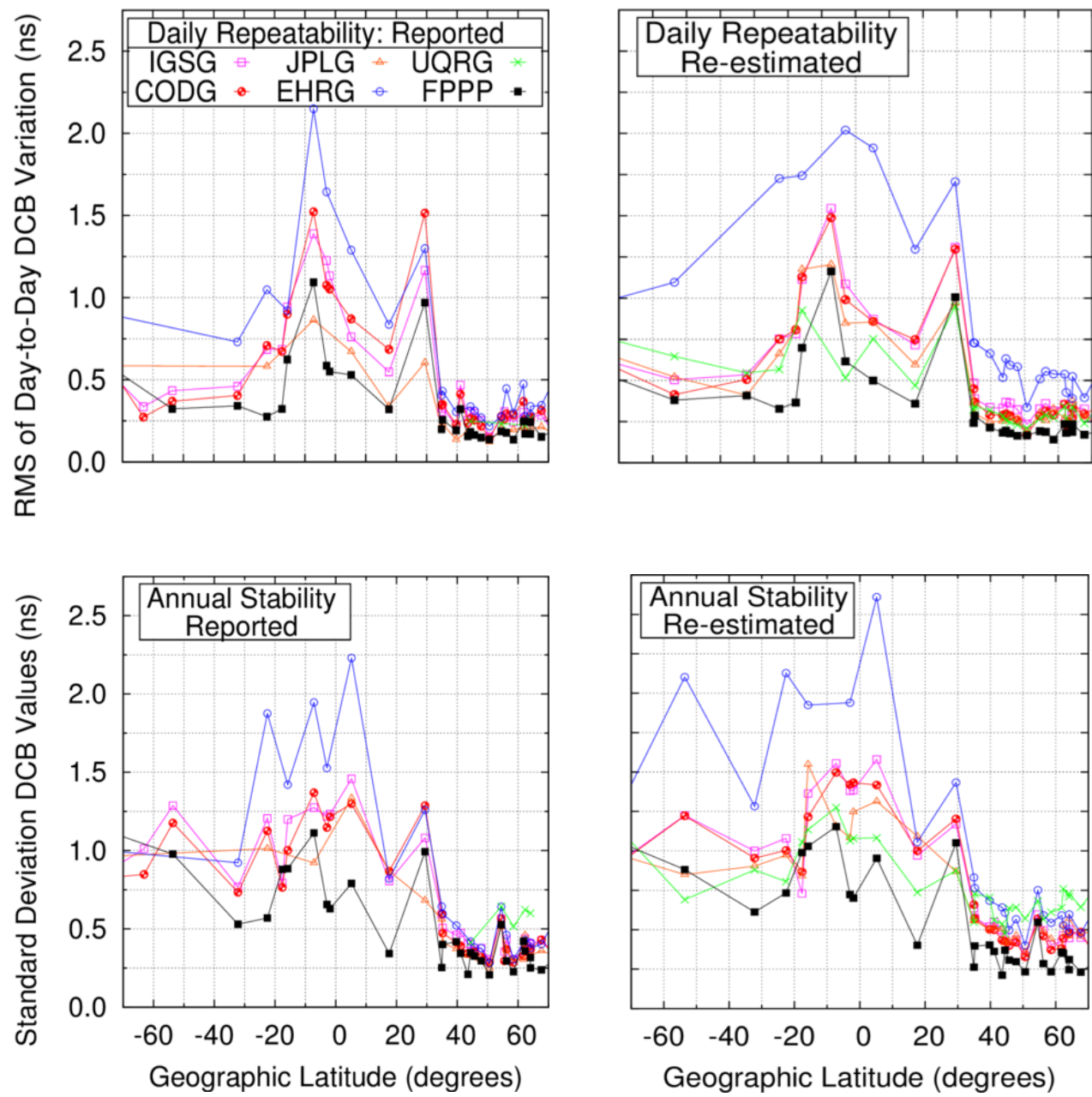

380

381 Fig. 7: Daily Repeatability (top) and Annual Stability (bottom) of receiver DCB estimates for 2014. Each point in the panels corresponds to an individual receiver (Table 2). The left-hand panels show the reported DCBs, re-aligned with the method introduced in this research. The right-hand panels show the re-estimated DCBs. The labels correspond to the same products as in Figure 5. Note: only stations having DCBs for more than 300 days are depicted. 
391 Table 2: Four character identifier of IGS stations and coordinates associated with Figure 7.

\begin{tabular}{|c|c|c|}
\hline $\begin{array}{c}\text { Station } \\
\text { ID }\end{array}$ & $\begin{array}{c}\text { Lat. } \\
\text { (deg.) }\end{array}$ & $\begin{array}{c}\text { Lon. } \\
\text { (deg.) }\end{array}$ \\
\hline RIO2 & $-53,60$ & $-67,75$ \\
\hline SUTM & $-32,21$ & 20,81 \\
\hline CHPI & $-22,55$ & $-44,99$ \\
\hline SCRZ & $-17,69$ & $-63,16$ \\
\hline STHL & $-15,84$ & $-5,67$ \\
\hline DGAR & $-7,22$ & 72,37 \\
\hline MAL2 & $-2,98$ & 40,19 \\
\hline KOUR & 5,22 & $-52,81$ \\
\hline CRO1 & 17,65 & $-64,58$ \\
\hline
\end{tabular}

\begin{tabular}{|c|c|c|}
\hline $\begin{array}{c}\text { Station } \\
\text { ID }\end{array}$ & $\begin{array}{c}\text { Lat. } \\
\text { (deg.) }\end{array}$ & $\begin{array}{c}\text { Lon. } \\
\text { (deg.) }\end{array}$ \\
\hline LHAZ & 29,49 & 91,10 \\
\hline NURK & 30,09 & $-1,93$ \\
\hline NICO & 34,96 & 33,40 \\
\hline GOLD & 35,24 & $-116,89$ \\
\hline QUIN & 39,79 & $-120,94$ \\
\hline ZECK & 43,60 & 41,57 \\
\hline HLFX & 44,49 & $-63,61$ \\
\hline FRDN & 45,74 & $-66,66$ \\
\hline VALD & 47,91 & $-77,56$ \\
\hline
\end{tabular}

\begin{tabular}{|c|c|c|}
\hline $\begin{array}{c}\text { Station } \\
\text { ID }\end{array}$ & $\begin{array}{c}\text { Lat. } \\
\text { (deg.) }\end{array}$ & $\begin{array}{c}\text { Lon. } \\
\text { (deg.) }\end{array}$ \\
\hline HERS & 50,68 & 0,34 \\
\hline FLIN & 54,54 & $-101,98$ \\
\hline ARTU & 56,25 & 58,56 \\
\hline CHUR & 58,59 & $-94,09$ \\
\hline YAKT & 61,87 & 129,68 \\
\hline YELL & 62,32 & $-114,48$ \\
\hline HOFN & 64,12 & $-15,20$ \\
\hline BAKE & 64,17 & $-96,00$ \\
\hline KIRU & 67,72 & 20,97 \\
\hline
\end{tabular}

Table 3: Mean values of Daily Repeatability and Annual Stability of re-estimated DCBs for receivers above $30^{\circ}$ North in Figure 7 (right column panels). The values are in nanoseconds.

\begin{tabular}{|c|c|c|c|c|c|c|}
\cline { 2 - 7 } \multicolumn{1}{c|}{} & IGSG & CODG & JPLG & EHRG & UQRG & FPPP \\
\hline $\begin{array}{c}\text { Mean Daily } \\
\text { Repeatability }\end{array}$ & 0.32 & 0.29 & 0.30 & 0.51 & 0.29 & 0.20 \\
\hline $\begin{array}{c}\text { Mean Annual } \\
\text { Stability }\end{array}$ & 0.48 & 0.47 & 0.49 & 0.58 & 0.67 & 0.33 \\
\hline
\end{tabular}

Relating Figure 7 with Figure 5, the value observed in the top left panel of Figure 7 at the latitude $17.65 \mathrm{~N}$ is associated with the thickness of the pattern of the DCB estimates of station CRO1 over the year in Figure 5, i.e. RMS of day-to-day variation. Alternately, the value shown in the bottom left panel of Figure 7 at the latitude $17.65 \mathrm{~N}$ is associated with the pattern itself of the DCB estimates of station CRO1, i.e. the standard deviation of the time series.

A degradation of DCB Daily Repeatability and Annual Stability for low-latitude 405 receivers can be observed in all panels of Figure 7 . This degradation agrees with the large 
ionospheric error shown in the bottom panel of Figure 6 in this particular region. On the other hand, the DCB estimates for receivers in the northern hemisphere show improved performance, i.e. greater repeatability and stability, with regard to the southern hemisphere receivers. This enhancement occurs because the northern hemisphere is a well-sounded region, thanks to a large number of reference stations available (Figure 4 for satellite PRN02). This leads to better ionospheric sounding, which improves the performance of all ionospheric models, because of the higher decorrelation between the DCBs and ionosphere.

413 In contrast, in the southern hemisphere there are fewer stations available, which results in poor geometry and degrades the performances, as seen in Figure 7.

It is also noticeable that, except for the ESOC determinations, similar results are found when comparing the left and right panels of Figure 7. This means that these receiver DCBs absorb similar ionospheric mis-modeling in both approaches, the reported and the reestimated DCBs. The degradation in the performance of the ESOC re-estimated DCB, is due to the greater errors in the ionospheric model associated to these estimates. This worse ionospheric modeling can be seen in Figure 6 (top and bottom). This occurs despite having used the ESOC GIMs at 1 hour sampling rate.

Table 3 shows the mean value of Daily Repeatability and Annual Stability of the reestimated DCBs for the receivers located over $30^{\circ}$ North, i.e. the best sounded region. We selected these re-estimated determinations because, as commented before, these values are more traceable and homogeneous. Moreover, as we will show in next section for the satellite DCBs, they better bound the actual error. These results illustrate how Daily Repeatability and Annual Stability are improved by the better ionospheric modeling of Fast-PPP. In this case, the improvement is $30 \%$ and $40 \%$, respectively, relative to the IGS determinations.

Figure 8 is similar to Figure 7, but for the satellite DCBs that are shown as a function of SVN. The satellite Blocks are highlighted by background bands of gray (Block-IIA), yellow (Block-IIR) and white color (Block-IIF).

The effect of the ionospheric mis-modeling in the case of satellite DCBs is not as straightforward as with the receiver DCBs. As already shown in Figure 4, the ionospheric region sounded by the measurements used in the determination of satellite DCBs is larger than the region for the receiver DCBs. This is the reason why the Daily Repeatability and 
Annual Stability of the satellite DCB estimates is much better than for the receiver ones: a factor of 3 or 4 when the receiver is in the well-sounded region of the northern hemisphere, and larger in other regions. This lower estimation noise allows for the detection of smaller anomalies than in the case of receiver DCBs.

442
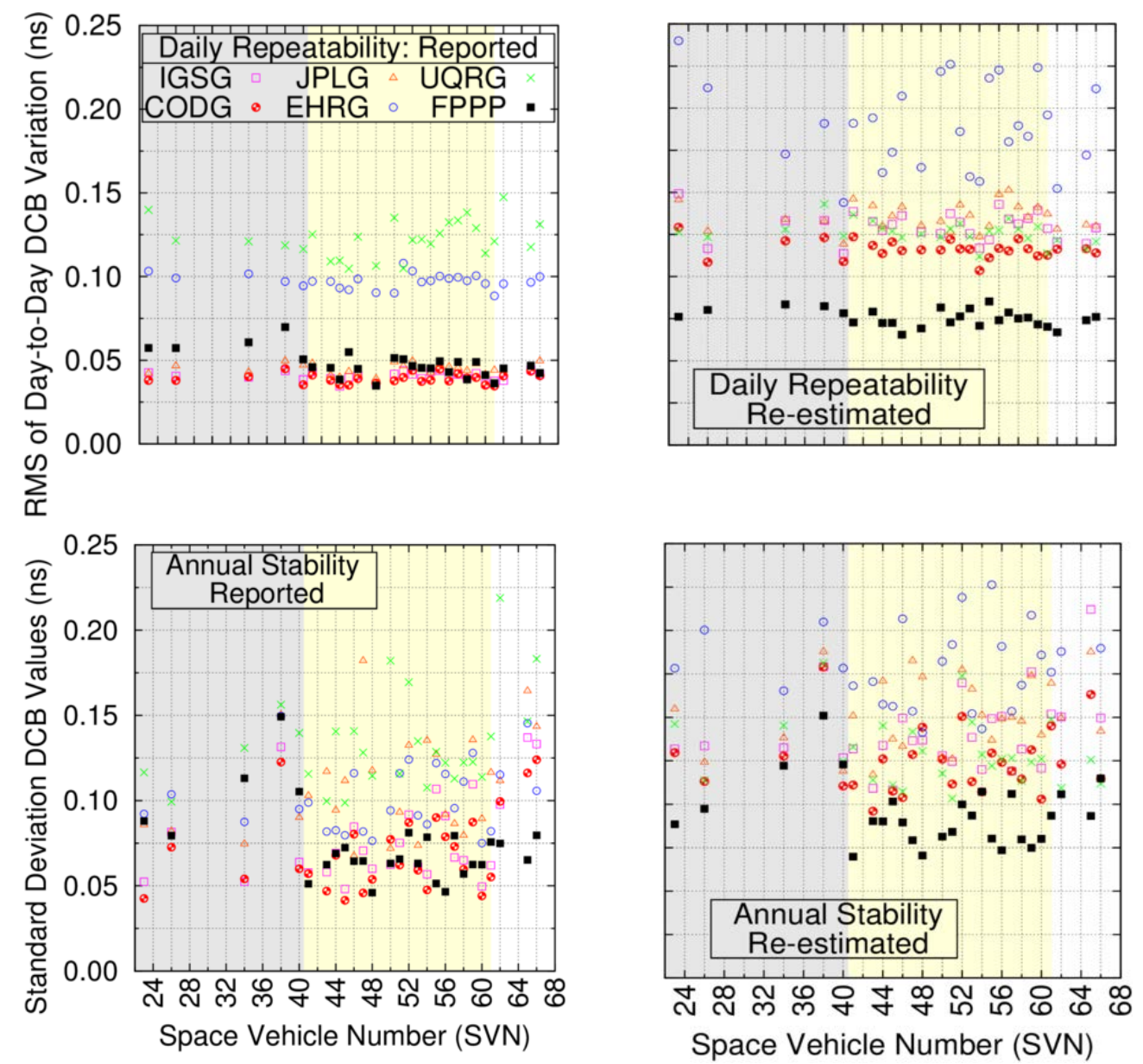

443

444 Fig. 8: Daily Repeatability of satellite DCB (top) and Annual Stability (bottom) estimates as 445 a function of SV number for 2014. The left panels show the reported DCBs, re-aligned with 446 the method introduced in this work. The right panels show the re-estimated DCBs. The labels correspond to the same products as in Figure 5. Background: gray (Block-IIA), yellow 448 (Block-IIR), white (Block-IIF).

As shown in Figure 8, all IAACs with exception of UPC have larger values in the reestimated DCBs (right panels) than in the reported ones (left panels). The results of UPC are quite similar in the left and right panels because, as commented before, UPC estimates the 
453 DCBs using the pre-computed GIM in a similar manner as used in the right panel 454 computations.

455 In the case of Fast-PPP, the repeatability of reported DCBs is slightly better than the re456 estimated ones. As noted in the introduction, this is because of the additional constraints, or 457 smoothing conditions, applied when computing the Fast-PPP GIM. These constraints are not 458 applied when estimating the DCBs (the reported ones) in the common adjustment process 459 with the ionosphere, which, as already mentioned, is done before applying the smoothing. 460 Thus, the previous results also suggest that most of the IAACs are applying some constraints 461 in the DCBs or in the ionosphere model. This effect is not seen in the receivers DCBs, because it is clearly under the accuracy of such estimates, i.e. it is at the level of $0.1 \mathrm{~ns}$. patterns than the Daily Repeatability. This is because the computation of the standard deviation of satellites DCBs throughout the full year is dominated by the seasonal mismodeling of the ionosphere. However, as in the upper panels, the right-hand bottom panel shows larger values, which can result from the constraints mentioned above.

\section{Block-IIA DCB satellites under eclipse conditions}

470 In Figure 8, mainly in the left panels, the larger values of Daily Repeatability and Annual 471 Stability exhibited by the Block-IIA satellites (gray shadow) for the Fast-PPP DCBs are 472 found.

This behavior is analyzed in more detail in Figure 9 for the DCB values of the GPS satellites PRN10 (SVN040, Block-IIA) (top panel), and PRN08 (SVN038, Block-IIA) (bottom panel). The figure depicts the reported DCB values from the IGSG and FPPP products as a function of time and for the entire year of 2014.

As can be observed in Figure 9, some peaks appear in the case of FPPP DCBs, which cannot be associated with ionospheric mis-modeling, but are related to the eclipse periods of these Block-IIA satellites, and are highlighted in the plots by the gray shadow. A similar behavior is also experienced by the other three satellites of Block-IIA. Thermal effects on hardware delays have been already reported by Yue et al. (2011) and Zhong et al. (2016) when studying the DCBs of a receiver on board of a Low Earth Orbiter (LEO) satellite. Such

483 effects are also in line with the thermally induced clock offset variations observed in 484 Montenbruck et al. (2011) for satellite SVN062 of Block-IIF. It is noteworthy that the peaks 
in Figure 9 appear even though the DCBs have been estimated as constant parameters in the Fast-PPP Central Processing Facility (CPF) and can be identified thanks to the accurate ionospheric modeling that allows for estimation of the satellite DCBs at the level of 0.05 ns of RMS Daily Repeatability (Figure 8, top left).

489
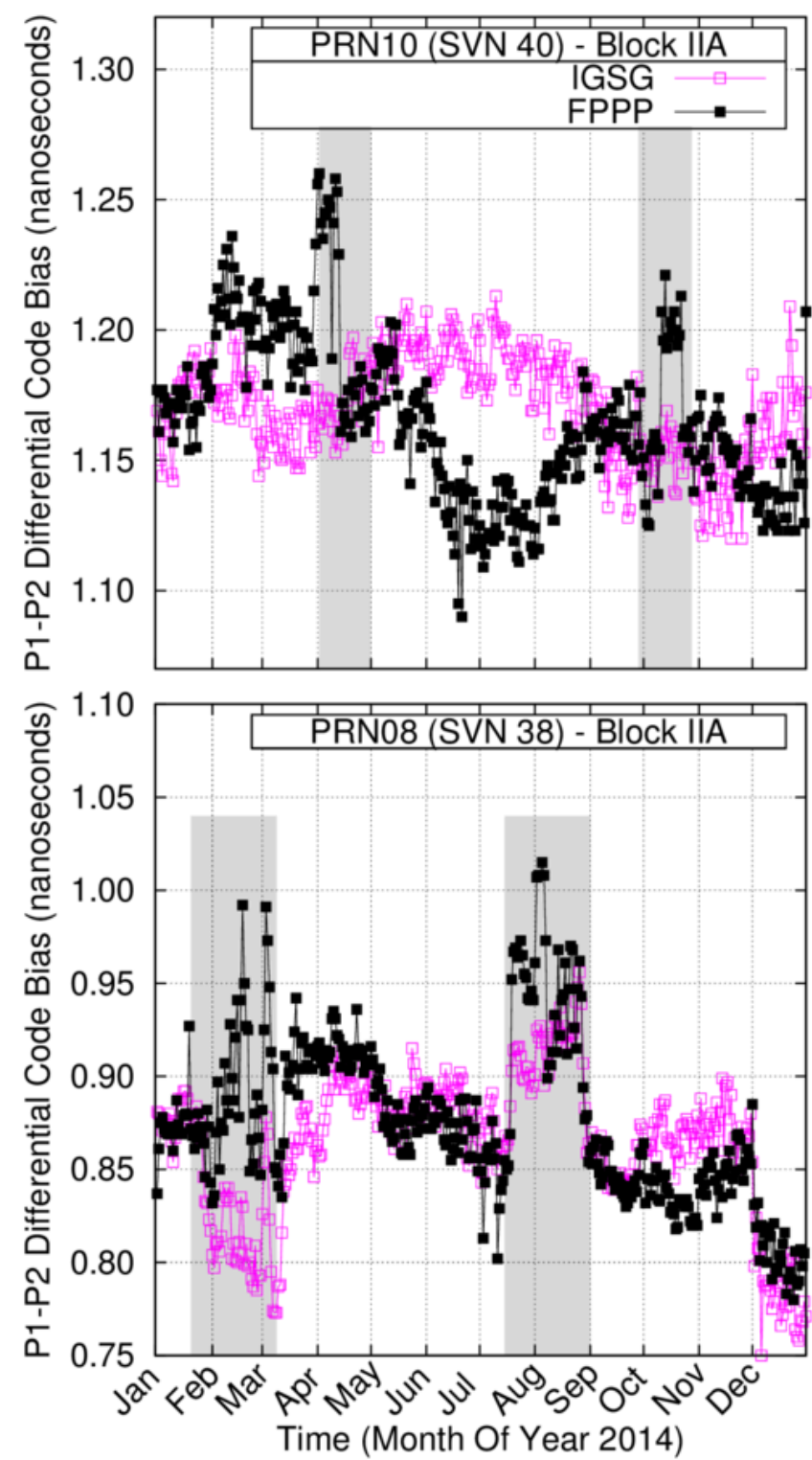

491

Fig. 9: Reported satellite DCB estimates as a function of time for the entire year of 2014: top PRN10 (SVN040, Block-IIA), bottom PRN08 (SVN038, Block-IIF). The estimates correspond to IGS combined Final Product (IGSG, pink squares) and Fast-PPP (FPPP, black squares). The gray bands indicate eclipse periods. 
494 Fast-PPP estimation procedure, the previous results indicate that i) the assumption that such

495 DCBs are "always" constant over time can introduce an error in their estimates and ii) as the 496 eclipse condition lasts for less than 2 hours, the peaks of up to 0.1 ns seen in the panels of 497 Figure 9 are actually part of a much longer oscillation, as a result of assuming that the DCB is 498 constant in the daily estimation. It is worth noting that such small effects can only be 499 observed with a highly accurate ionospheric model. The discontinuity observed by the 500 PRN08 (SVN 038) at November 21, 2014 occurred after NANU2014078 was issued, having 501 set the satellite unusable until further notice (http://celestrak.com/GPS/NANU/2014/ nanu.2014083.txt).

Finally, from the top panel of Figure 9, it follows that jumps around 0.05 ns cannot be detected by the IGSG products, while the Fast-PPP is sensitive to such variations. On the other hand, the bottom panel shows that a larger jump, at the level of $0.10 \mathrm{~ns}$, is detected by IGSG. These detection levels agree with the Daily Repeatability and Annual Stability figures of the re-estimated DCBs shown in the right panels of Figure 8, i.e. about 0.12-0.20 ns for the IAACs and about $0.7 \mathrm{~ns}$ for Fast-PPP. These results indicate that figures from the reestimated DCBs can be taken as a more realistic indicator of the accuracy of these products than the values linked to the reported ones, which seem rather optimistic.

\section{Summary and Conclusions}

513 The main results and findings of this work are as follows. A new method for the DCB 514 alignment has been proposed to remove the jumps and artificial drifts appearing in the 515 conventional alignment procedure used by IGS due to satellite constellation changes. This 516 new method can be applied in real-time in a straightforward manner and regardless of constellation changes.

518 Once the alignment problem is fixed, it is possible to identify smaller effects in the 519 DCB estimates. In this manner, an assessment of the Daily Repeatability and Annual Stability 520 of the DCBs has been conducted by considering the reported DCB values provided by the 521 IAACs and the Fast-PPP. Moreover, the DCBs have been re-estimated using the 'single522 layer' pre-computed GIMs given in the associated IAACs IONEX files and the pre-computed 523 'two-layer' Fast-PPP GIM. 
Similar ionospheric mis-modeling effects, such as seasonal variations in the time series for 2014, are clearly seen in the reported and re-estimated receiver DCBs for the different IAACs. This concurrence is because all of these centers are using the one-layer ionospheric model to de-correlate the ionosphere from DCBs, which is the main error source affecting these determinations. In contrast, the DCBs estimated with the two-layer Fast-PPP ionosphere show improved Daily Repeatability and Annual Stability because, among other aspects of the processing strategy, the Fast-PPP model is able to accommodate the variation in the vertical distribution of the electron content due to such diurnal or seasonal effects, see for instance Rovira-Garcia et al. (2016).

In general, the reported DCBs show smoother values, i.e. higher repeatability, than the re-estimated ones. The smoothed values in the reported DCBs are probably produced by constraints introduced by the IAACs in the DCBs or ionospheric model in the common adjustment process of these two determinations. The satellite DCBs re-estimated using the pre-computed Fast-PPP GIM depict only slightly worse repeatability and stability values than those computed by the joint estimation of DCBs and ionosphere, i.e. the reported DCBs. This worsening is due to constraints imposed on the ionosphere when filling the data gaps, e.g. over oceans, to build up the Fast-PPP GIM.

Finally, DCB variations at the level of 0.1 ns have been observed in the Fast-PPP estimates for Block-IIA satellites under eclipse conditions. These variations are likely due to changes in the satellite temperature. In the case of the IAAC estimates, such variations are only perceptible in satellite PRN08 (SVN038) because they are approximately two times larger, i.e. at the level of 0.2 ns. This result suggests that the accuracy of the DCB estimates is more related to the repeatability found in the re-estimated values than to the values given in the IONEX files, i.e. the reported ones. Then, assuming this fact, we can conclude that the accuracy of the reported receiver DCBs is approximately 0.3-0.5 ns for the IAAC estimates and 0.2 ns for the Fast-PPP ones. For the satellite DCBs, these values are between 0.12-0.20 ns for IAACs and 0.07 ns for Fast-PPP. 
557 The authors acknowledge the use of data and products from the International GNSS Service.

558 This work has been partially sponsored by the Spanish Ministry of Science and Innovation project CGL2015-66410-P, the ESA/ESTEC ICASES project PO 1520026618/01, and the ESA/EPO project EG-SIFE, Contract No. 40001122/14/NL/WE.

561

562

563

\section{References}

564 Colleen HY, Feess WA, Esposti RD, Chasko A, Cosentino B, Wilson B, Wheaton B (1999) GPS Satellite Interfrequency Biases. Proc. of ION GPS 1999, Institute of Navigation, Cambridge, MA, USA, June 27 - 30. pp. 347-354.

567

568

569

570

571

572

573

574

575

576

577

578

579

580

581

582

583

584

Hernández-Pajares M, Juan JM, Sanz J, (1999) New approaches in global ionospheric determination using ground GPS data. J of Atmospheric and Solar Terrestrial Physics, 61:1237-1247 doi: 10.1016/S1364-6826(99)00054-1.

Hernández-Pajares M, Juan JM, Sanz J, Orus R, Garcia-Rigo A, Feltens J, Komjathy A, Schaer SC, Krankowski A (2009) The IGS VTEC maps: A reliable source of ionospheric information since 1998. J. Geod, 83:263-275, doi: 10.1007/ s00190-008-0266-1.

Juan, JM, Rius A, Hernández-Pajares M, Sanz J (1997) A two layers model of the ionosphere using Global Positioning System data. Geophys Res Lett, 24(4):393-396. doi: 10.1029/97GL00092.

IS-GPS-200H (2014). Navstar GPS Space Segment/Navigation User Interface. IS-GPS-200H Interface Control Document, see http://www.gps.gov/technical/icwg/IS-GPS-200H.pdf

Komjathy A, Wilson BD, Runge TF, Boulat BM, Mannucci AJ, Sparks L, Reyes MJ (2002) A New Ionospheric Model for Wide Area Differential GPS: The Multiple Shell Approach. Proc. ION NTM 2002, Institute of Navigation, San Diego, CA, USA, January 28-30, 460-466.

Li Z, Yuan Y, Li H, Ou J, Huo X (2012) Two-step method for the determination of the differential code biases of COMPASS satellites. J Geod. 86(11):1059-107. doi: 10.1007/s00190-012-0565-4. 
606

607

608

609

610

611

612

613

614

615

616

Montenbruck O, Hugentobler U, Dach R, Steigenberger P, Hauschild A (2011) Apparent clock variations of the Block IIF-1 (SVN062) GPS satellite, GPS Solut. 16(3):303-313 doi: 10.1007/s10291-011-0232-x.

Montenbruck O, Hauschild A, Steigenberger P (2014) Differential code bias estimation using multi-GNSS observations and global ionosphere maps. Navigation 61(3):191-201. doi: 10.1002/navi.64.

Ray J, Senior K (2005) Geodetic techniques for time and frequency comparisons using GPS phase and code measurements. Metrologia 4(42):215-232. doi: 10.1088/00261394/42/4/005.

Rovira-Garcia A, Juan JM, Sanz J, González-Casado G (2015) A World-Wide Ionospheric Model for Fast Precise Point Positioning IEEE Trans Geosci Remote Sens, 53(8):45964604, doi:10.1109/TGRS.2015.2402598.

Rovira-Garcia A, Juan JM, Sanz J, González-Casado G, Ibáñez-Segura D (2016) Accuracy of ionospheric models used in GNSS and SBAS: methodology and analysis. J Geod. 90(3):229-240. doi: 10.1007/s00190-015-0868-3.

Schaer S, Guenter W, Feltens J (1998) IONEX: The IONosphere Map EXchange Format Version 1. https://igscb.jpl.nasa.gov/ igscb/data format/ionex1.pdf.

Schaer S (2008) Differential Code Biases (DCB) in GNSS Analysis. Proceedings of International GNSS Service Analysis Center Workshop 2008, Miami Beach. https://www.ngs.noaa.gov/IGSWorkshop2008/docs/Schaer_DCB_IGSWS2008.ppt

Yue, X, Schreiner WS, Hunt DC, Rocken C, Kuo YH (2011) Quantitative evaluation of the low Earth orbit satellite based slant total electron content determination. Space Weather 9(9), S09001. doi: 10.1029/ 2011SW000687.

Zhang D, Shi H, Jin Y, Zhang W, Hao Y, Xiao Z (2014) The variation of the estimated GPS instrumental bias and its possible connection with ionospheric variability. Sci. China Technol. Sci. 57(1):67-79. doi: 10.1007/s11431-013-5419-7.

Zhong J, Lei J, Dou X, Yue X (2016) Is the long-term variation of the estimated GPS differential code biases associated with ionospheric variability? GPS Solut 20(3):313-319. doi: 10.1007/ s10291-015-0437-5. 


\section{Biographies}

618

619 Jaume Sanz has been with the Department of Mathematics, Universitat Politecnica de 620 Catalunya (UPC), Barcelona, Spain since 1983. He was granted tenure and promoted to 621 Associate Professor in 1988. He obtained the National Accreditation for Full Professor in 622 2011. He has published over 70 papers in peer-reviewed journals and more than 200 works in 623 meeting proceedings, with four best paper awards from the U.S. Institute of Navigation. He is 624 a coauthor of five patents on GNSS and four books on GNSS Data Processing.

625

626 José Miguel Juan Since 1988, he has been with the Department of Physics, Universitat 627 Politecnica de Catalunya (UPC), Barcelona, Spain. He was granted tenure and promoted to 628 Associate Professor in 1991. He obtained the National Accreditation for Full Professor in 629 2011. He has published over 70 papers in peer-reviewed journals and more than 200 works in 630 Meeting proceedings, with four best paper awards from the US Institute of Navigation. He is 631 a coauthor of five patents on GNSS and four books on GNSS Data Processing.

632

633 Adrià Rovira-Garcia is a post-doctoral researcher at the Technical University of Catalonia 634 (UPC) and senior research engineer in the Research Group of Astronomy and Geomatics 635 (gAGE). He co-authors 6 papers in peer-reviewed journals, 2 book chapters and over 20 636 works in meeting proceedings, with 1 best presentation award from the US Institute of 637 Navigation and 1 Outstanding Poster Award from the European Geosciences Union.

639 Guillermo González-Casado is with the Department of Mathematics, Universitat Politecnica 640 de Catalunya (UPC), Barcelona, Spain, being granted tenure and promoted to Associate 641 Professor in 1999. His current research interests are focused in ionospheric modeling based in 642 GNSS observations and radio occultations, Ground-Based Augmentation Systems, and the 643 study and development of GNSS applications in general. He has published about 20 papers in 644 peer-reviewed journals and more than 25 works in meeting proceedings. 